\title{
Design and Implementation for Trucks Tracking System Using GPS Based on Semantic Web
}

\author{
Yasser A. Nada \\ Faculty of Computers and IT, Department of Computer Science, \\ Taif University, KSA
}

\section{ABSTRACT}

This paper proposed design and implementation of trucks tracking system using GPS based on semantic web for vehicle tracking in real time. In-vehicle unit and a tracking server is used. The information is transmitted to tracking server using GSM/GPRS modem on GSM network by using SMS or using direct TCP/IP connection with tracking server through GPRS. The received SMS contain longitude and latitude that are used to locate the vehicle on the Google maps. Tracking server also has GSM/GPRS modem that receives vehicle location information via GSM network and stores this information in database. It has been explored that most of the GPS based tracking systems are user friendly, cost effective, reliable and can be easily validated through different experiments and simulations which means that tracking system will send notifications periodically to the main station about the current position of the vehicle. Moreover those tracking notifications (GPS) will be stored in the database for future revising or generating required reports.. The tracking system will be better for cars, trucks, trailers, railways, containers and boats that can be traced using GPS vehicle tracking.

KEYWORD: Trucks Tracking System, Transportation, GPS, Semantic Web

\section{INTRODUCTION}

The vehicle tracking system is an electronic device that tracks the vehicle's location. Most of the tracking systems use the Global Positioning System GPS module to locate vehicles position. Many systems also combine communication components such as satellite transmitters to communicate the vehicle's location to the remote user. Google maps are used to view vehicles' location. The design of the tracking system has been divided into three parts: basic design, intermediate design and advanced design[1].
The GPS-GPRS-based tracking system is a system that makes use of GPS to determine the precise location of a vehicle to which the device is attached. When a large number of objects or vehicles are spread all over the ground, the owner of corporation needs to keep track for fuel saving, security purposes...etc. A tracking system is required to determine the location of any object at any given time and the distance travelled. Also, the need for a tracking system in users vehicle is used to prevent any kind of theft since police can use tracking reports to locate a stolen vehicle location. GPRS and GPS based tracking system will provide effective, real time vehicle location report[2].

A GPS-GPRS based tracking system gives all the specifications about the location of a vehicle. The system utilizes geographic position and time information from the Global Positioning Satellites $[3,4]$. This system is built based on embedded system, used for tracking and positioning of any vehicle by using Global Positioning System (GPS) and Global system for mobile communication (GSM). This design will continuously watch a moving Vehicle and report the status of the Vehicle on demand.

The market for GPS vehicle tracking systems is considered as one of the fastest growing markets for GPS applications. There are many levels of sophistication, but what all systems have in common is a GPS receiver and software to put the tracking results on a map. The differences in mapping programs are huge and it is very difficult to judge in advance how well these software solutions will perform.

In today's fast moving life GPS based tracking system has great importance in everyone's life where loved ones, vehicles, mobile phones and other devices can be located with a touch of a button. With the evolution in processing power, and information fed 
into the memory, for example, points of interest, road guides, topographic data, and many more, GPS receivers have the ability to change the location, speed, and time data into a helpful display design. GPS information might likewise be fed to a website for real-time location mapping [5].

Google Maps is a web mapping service developed by Google. It offers satellite imagery, street maps, $360^{\circ}$ panoramic views of streets (Street View), real-time traffic conditions (Google Traffic), and route planning for traveling by foot, car, bicycle (in beta), or public transportation. Like many other Google web applications, Google Maps uses JavaScript extensively [6]. Two main categories of GPS car tracking solutions are the passive systems and the real time systems. Passive systems store the gathered data in their internal memory and the owner has only access to the data when the unit returns to the base. Real time systems send the data at regular intervals to the manufacturer database and the user can read these through a website. Other systems can deliver data directly to the user cell phone on demand.

In this paper, it's intended to implement a tracking system for trucks that use the real time, which means tracking system will send notifications periodically to the main station about the current position. Moreover those tracking notifications (GPS) will be stored in the database for future revising or generating required reports.

\section{RELATED WORKS}

In this paper[1], we have proposed a novel method of vehicle tracking and locking systems used to track the theft vehicle by using GPS and GSM technology. This system puts into the sleeping mode vehicle handled by the owner or authorized persons; otherwise goes to active mode. The mode of operations changed by persons or remotely. When the theft identified, the responsible people send SMS to the micro controller, then issue the control signals to stop the engine motor. After that all the doors locked. To open the doors or to restart the engine authorized person needs to enter the passwords. In this method, easily track the vehicle place and door is locked.

Our system [7] decomposition allows each of the subsystems to be reusable by a wide variety of sensor network applications. The network management and debugging services are useful for deploying other sensor. We demonstrate a working system that not only monitors sensory data but also tracks and controls a higher tier system to accomplish a cooperative task in real time. The system assumes very little processing and communication requirements on the sensor. Logs of Tracking Server and Pointing out current location of vehicle. For vehicle tracking in real time, in-vehicle unit and a tracking server is used. The information is transmitted to Tracking server using GSM/GPRS modem on GSM network by using SMS or using direct TCP/IP connection with Tracking server through GPRS. Tracking server also has GSM/GPRS modem that receives vehicle location information via GSM network and stores this information in database.

In this research paper [5], quality assurance standards considered which are followed by different techniques used in systems in the surveyed papers. Upon quality standards these techniques are adopted to achieve Quality in their processes. Mainly in our survey all the techniques and methodologies used to provide secure, robust and low cost systems for the owners to satisfy their needs for tracking through GPS and GPRS of GSM network. Most of the systems used GSM network for the sending messages as it is the cheapest way to send SMS as compared to other modes of communication. Along with GSM technique, systems used web services to facilitate their users with the help of interactive web pages. Some systems follow the quality standards to improve their work with the growing need of security and accuracy in the services but some are unable to compete with them in this quality standard. Now days, achieving quality is very important to compete with others in this growing technological world to uphold your work in international market and to fulfill the customer requirements. For future work, more services could be added to the mobile application, make it more fast and accurate, and also the graphical user interface could be improved in these systems to make it more interactive for the users.

The vehicle tracking system [8] presented in this paper can be used for positioning and navigating the vehicle with an accuracy of $10 \mathrm{~m}$. The positioning is done in the form of latitude and longitude along with the exact location of the place, by making use of Google maps. The system tracks the location of a particular vehicle on the user's request and responds to the user via SMS. The received SMS contains longitude and latitude that is used to locate the vehicle 
on the Google maps. The vehicle tracking system allows a user to: remotely switch $\mathrm{ON}$ the vehicle's ignition system, remotely switch OFF the vehicle's ignition system, remotely lock the doors of the vehicle, remotely unlock the doors of the vehicle, and remotely track a vehicle's location. Some changes were made in which most notable change was alteration of the tracking methodology (i.e. Access to 32 channels of satellites instead of 3 ). The vehicle tracking system was built successfully. However, the vehicle tracking system could be made more robust by using more accurate GPS unit.

\section{SYSTEM REQUIREMENTS}

The overall system functionality comes from interaction between the system components which are:

- GPS receiver tool

- Web application and purpose designed database

- VB.Net

○ SQL Server

- Flash (Google Maps)

\section{A. Functional Requirements}

Functional requirements capture the intended behavior of the system. This behavior may be expressed as services, tasks or functions which the system is required to perform.

Descript the activity and services a system must provide. Inputs, outputs, processes, stored data

○ There will be two types of users: Drivers Manager

- Manager should be able to view Maps

- Manager should be able to track Trucks and Drivers

- Manager can manage all trucks to access GPS signals history and received data

\section{B. XML}

Extensible Markup Language (XML) is a set of rules for encoding documents in machine-readable form. The design goals of XML emphasize simplicity, generality, and usability over the Internet. It is a textual data format with strong support via Unicode for the languages of the world.
These supporting technologies include the Web, Web databases, information retrieval, metadata, information management, and electronic commerce (e-commerce). Figure (1) illustrates the Applications of XML.

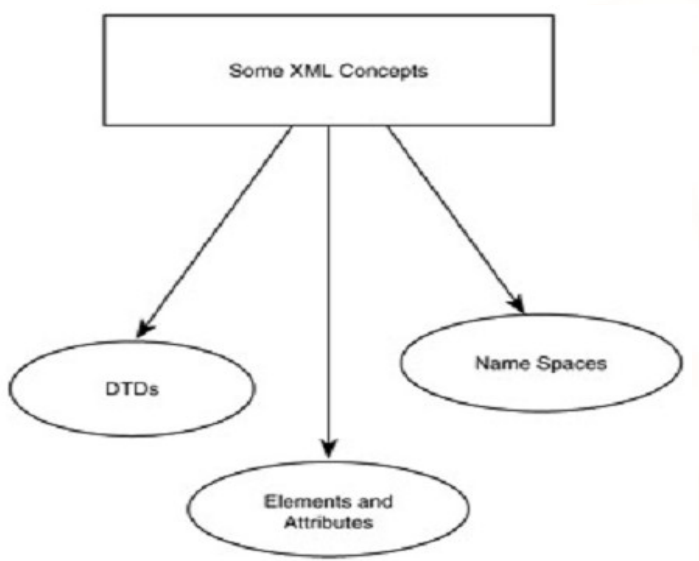

Figure 1: Applications of XML

A core data representation format for semantic web is Resource Description Framework (RDF). RDF is a framework for representing information about resources in a graph form. It was primarily intended for representing metadata about WWW resources, such as the title, author, and modification date of a Web page, but it can be used for storing any other data. It is based on triples subject-predicate-object that form graph of data. All data in the semantic web use $\mathrm{RDF}$ as the primary representation language. The normative syntax for serializing RDF is XML in the $\mathrm{RDF} / \mathrm{XML}$ form [9]. Formal semantics of $\mathrm{RDF}$ is defined as well in Figure 2.

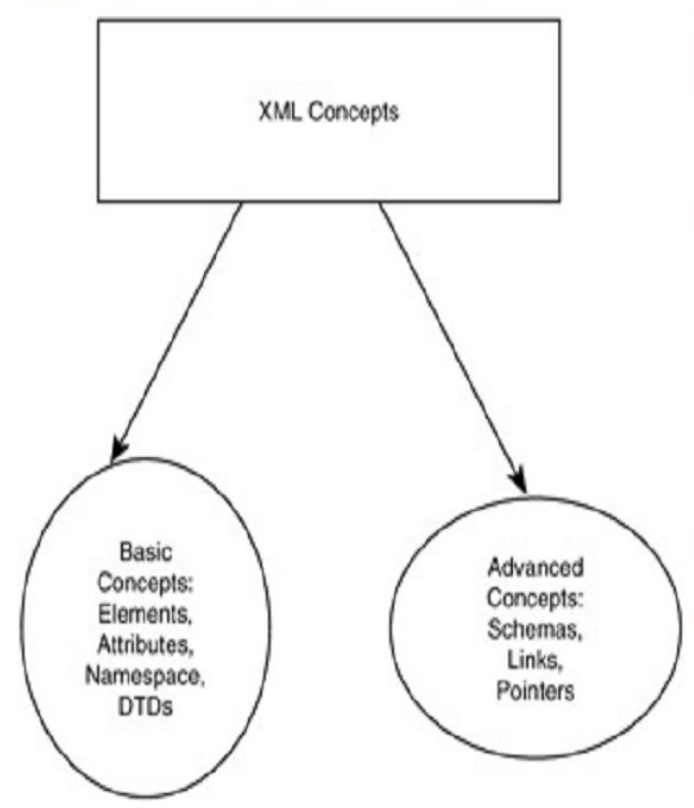

Figure 2: XML concepts 


\section{VISUAL BASIC.NET}

VB.Net will be used as the main language in this paper, because it is building a system that needs screens and tables to store in the database.

The system as programming will be divided in two parts, the first part which will be installed on the PC's inside the trucks, that part works as a receiver for the GPS signals and send data when requested from the second part of the software which is the administration. The administration will receive the signal and see it on the map, and if any location may be reflected by that signal, the system will give that on map.

\section{SQL SERVER}

In this paper, an SQL Server is used to store data, data about trucks, about drivers, about GPS locations which are taken and must be stored for future reference. Moreover, the maps and locations are stored; also required locations must be stored to be tested periodically. Since the researcher needs a huge storage of data, for example the database will store the GPS records for tracks for several months or maybe years. That directed us to use the most useful and powerful database: SQL Server.

\section{E. FLASH (GOOGLE MAPS)}

Flash from Google maps is used to implement Maps for example, if certain important location in one place the map will be show in flash the track to that position, a moving red track that will be obvious and clear. Flash is making a prominent presence into the web world these days. Flash is the best medium to put animated and interactive movies onto websites to catch eyes instantly.

\section{F. GSM/GPS MODULE}

Quad-Band SIM908 module is used which combines GPS technology for satellite navigation with worldwide known technology GSM. This module is configured to connect to navigation satellite and get GPS location at predetermined intervals and send this information to web application through GPRS service provided by GSM [2].

\section{DRAW FLOW DIAGRAM (DFD) CONTEXT DIAGRAM}

That diagram summarizes the data flowing inside the proposed system that shows the two types of users:
Driver and Manager, and shows all the incoming and outgoing data.

The driver could receive his current location using the GPS Receiver tool, and also send a location to the station to be viewed by the manager.

The manager could manage all maps, trucks, drivers and locations inside the system, and sure could receive all GPS readings (in Figure 3).

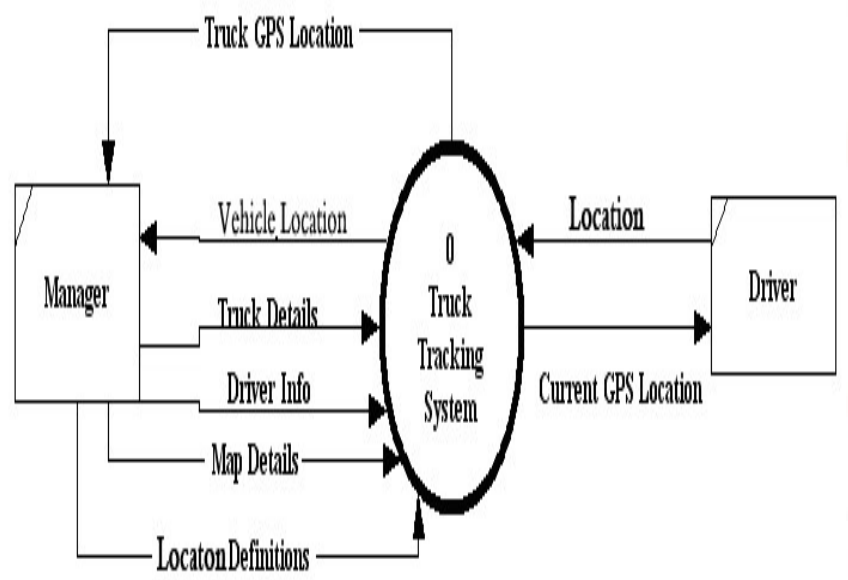

Figure 3: Context Diagram

\section{A. DFD LEVEL 0}

That diagram describes and explains in detail the processes that are performed inside the system. There will be sequence of processes each of them reflects one of the authorities for each user. Each process has certain number to define. Also, the data stores which reflect the data tables in the proposed system (in Figure 4)

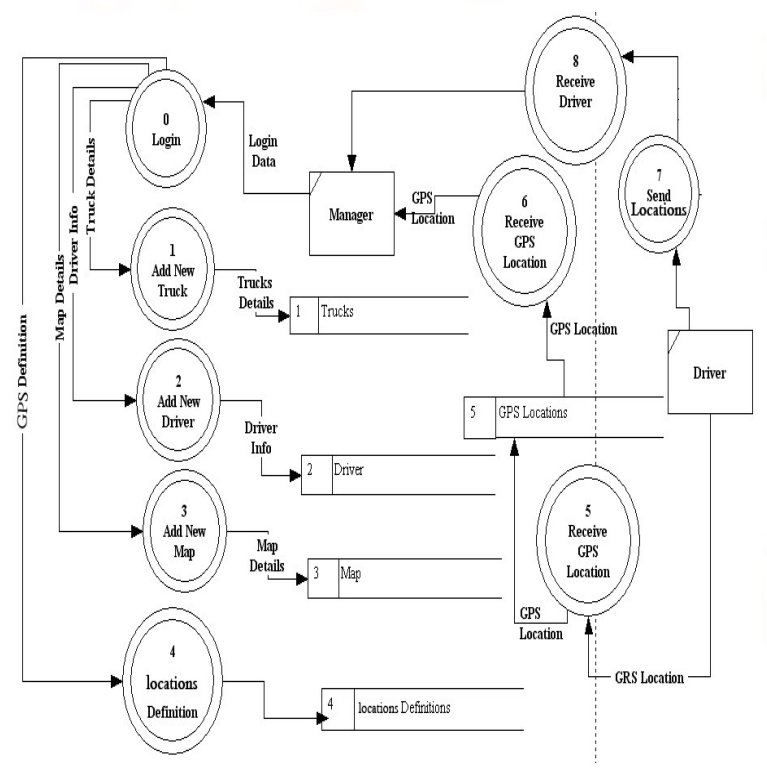

Figure 4: Level 0 Diagram 


\section{B. ER DIAGRAM (ENTITY RELATIONSHIP DIAGRAM)}

That diagram shows the main entities in the software and links entity relationship diagram between them. There will be seven entities and all are connected to each other through primary key in each table. Those tables will store data to generate reports also (in Figure5).

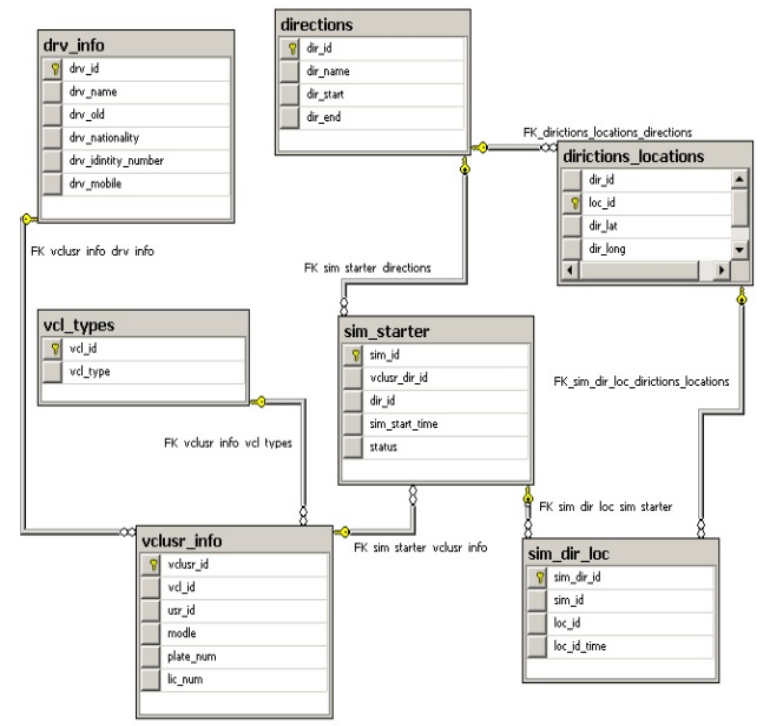

Figure 5: ER Diagram

\section{DATABASE DESIGN}

Normalized database tables use results in reduction in cost as the redundancy is avoided as much as possible. Repetition when occurs exhausts the server and database engines by checking similar data existing in a number of different tables. Using the standardized set procedures and distributing database functionality into set of stored procedures reduce the needed code amount and syntax lines of code used [10].

As mentioned above there will be seven tables implemented using SQL server 2005 as follow (in Figure 6, 7).

- Trucks Drivers Table:

\begin{tabular}{l|l|l|l|}
\multicolumn{1}{|c|}{ Column Name } & \multicolumn{1}{|c|}{ Data Type } & Allow Nulls \\
\hline velusr_id & int & $\Gamma$ \\
\hline vol_id & int & $\Gamma$ \\
\hline usr_id & int & $\Gamma$ \\
\hline modle & nvarchar(50) & $\Gamma$ \\
\hline plate_num & nvarchar(50) & $\Gamma$ \\
\hline
\end{tabular}

Figure 6: Trucks Drivers Table
- GPS Maps Table:

\begin{tabular}{|c|c|c|c|}
\hline & Column Name & Data Type & Allow Nulls \\
\hline D & dir_id & int & $\Gamma$ \\
\hline i & loc_id & int & г \\
\hline & dir_lat & nvarchar(50) & Г \\
\hline & dir_long & nvarchar(50) & Г \\
\hline
\end{tabular}

Figure 7: GPS Maps Table

\section{DESIGN SEMANTIC WEB TRUCKS TRACKING SYSTEM}

The Semantic Web is a web that is able to describe things in a way that computers can understand. In ontology technology classes and their instances are used to describe domain concept specific resources, data properties and object properties are used to describe characteristics of resources and relationships between them [10]. A challenge for the Semantic web is enabling information interoperability between related but heterogeneous ontology [11].

The Semantic Web trucks tracking system is a system designed using a combination of several modern information and communications technologies. The system comprises of Trucks tracking devices, a central server system and a web-based application. The web based system enables user to browse location track on map through developed web application embed Google Map and interact with database server for vehicles track details.

The trucks tracking system is shown in Figure 8. System contains a GPS, and GSM modem and overall system reside into a truck. A tracking system will provide effective real time vehicle location reporting. Tracking system will inform where your truck is and where it has been, how longer it has been there. The GPS receiver of truck terminal receives and resolves the navigation message broadcasted by GPS position satellites, computes the longitude and latitude of truck coordinates, transforms it into the GSM message form by GSM communication controller, and sends the message to monitoring center via the GSM network. 


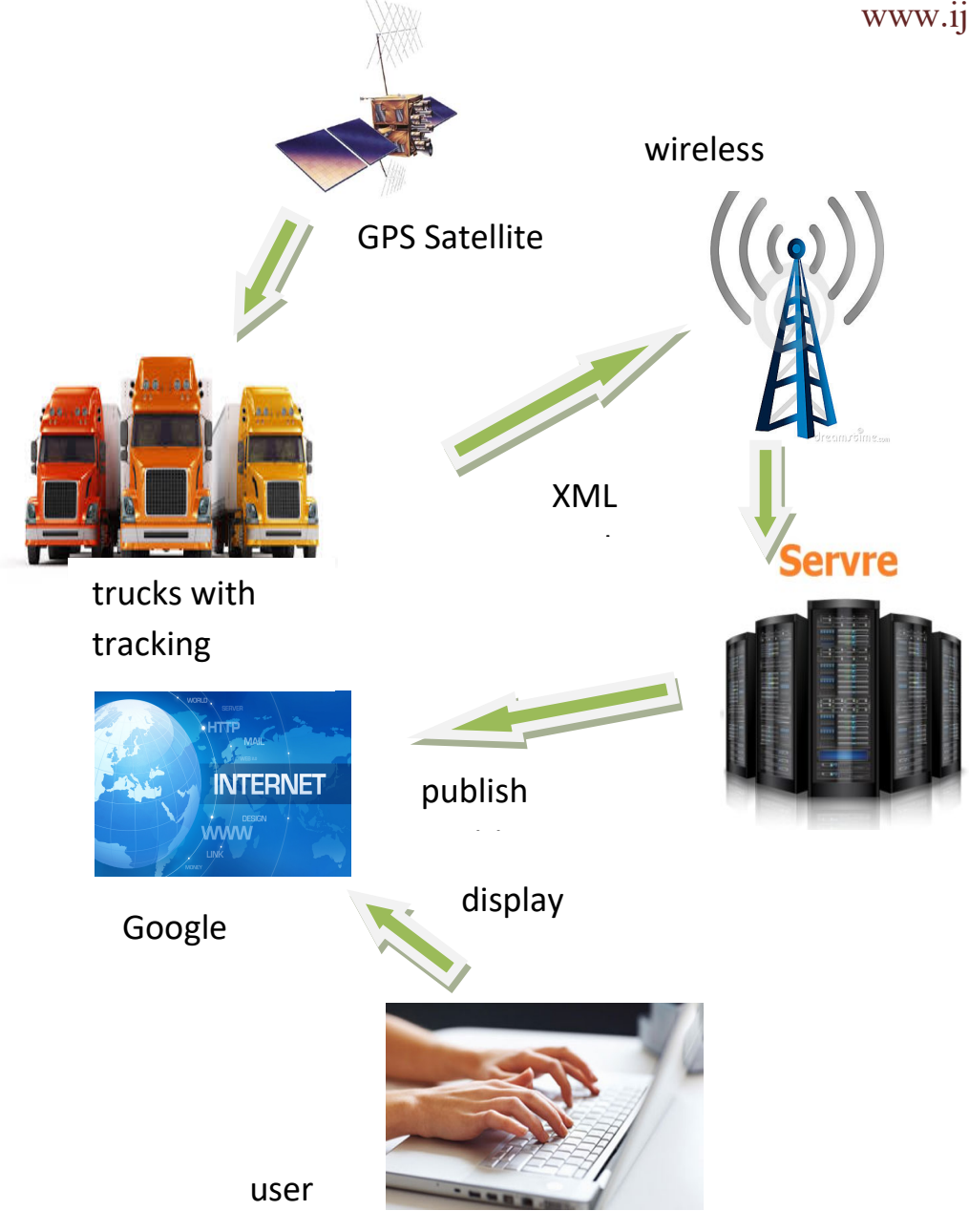

Figure 8: The Trucks Tracking System Overview

The location is acquisitioned from satellite using GPS receiver location coordination sent through GPRS, the GSM network will pass the information to the destination server as XML packet. The clients can browse track on electronic map using purpose designed web application on website.

\section{RESULT AND DISCUSSION}

Complete system as shown in fig.9, 10, 11, and 12 contains complete connection with GSM and GPS system. The positions are displayed in requesting cell phone display. With this system it becomes easy for the users to keep track of their objects. If the object resides in any location positional data in terms of latitude and longitude can easily be traced out.

Accuracy of the system is highly dependent on the GPS device and the coordinates received from GPS satellite while reliability and usability depend on the reliability of the mobile communications network.

Here we list the main screens as follows:

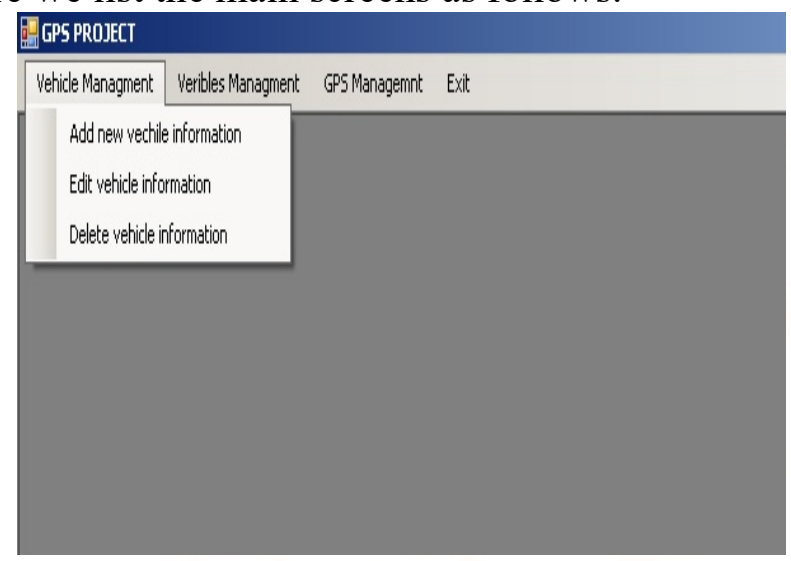

Figure 9: The Design of Vehicles Management Menu

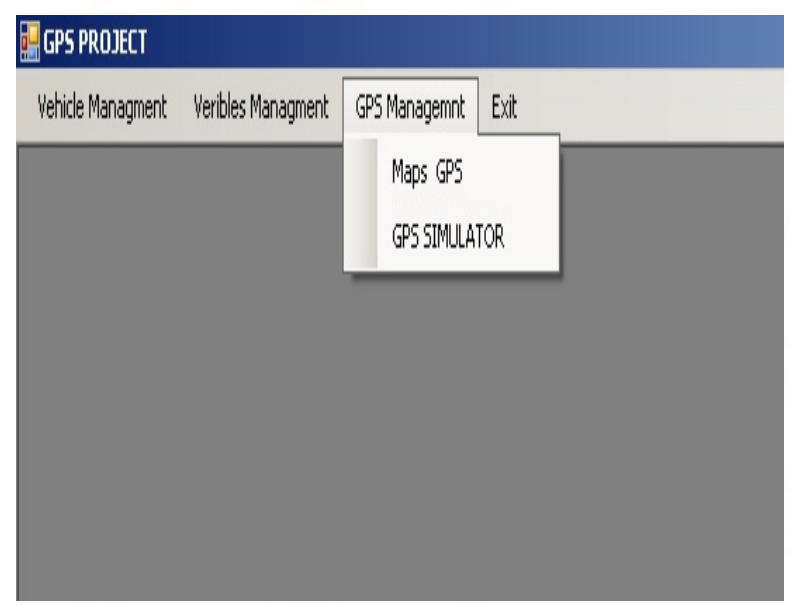

Figure 10: The Design of GPS Management Menu

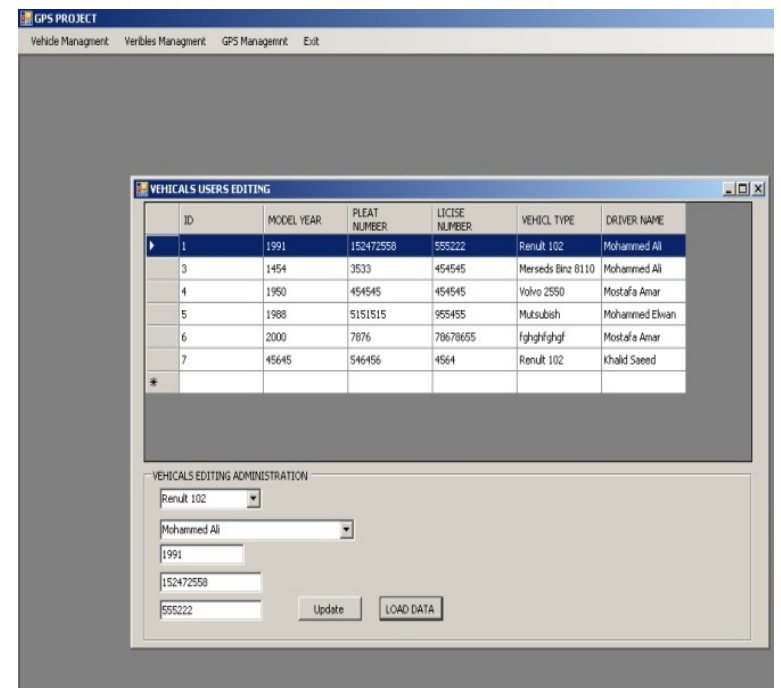

Figure 11: The Design of Editing Vehicles Related to Users Form 
The objective of the paper is to build an additional feature to the present security system that will warn the owner of the vehicle by sending SMS when there has been an intrusion into the vehicle. To provide a solution to avoid car stolen in the lower cost than advance security car system

The signals will be received by the station through the connection with the same server, since we will implement the tracking system as software. Google Maps provide zoom and moving tools which ease zooming in and out and navigation on map.

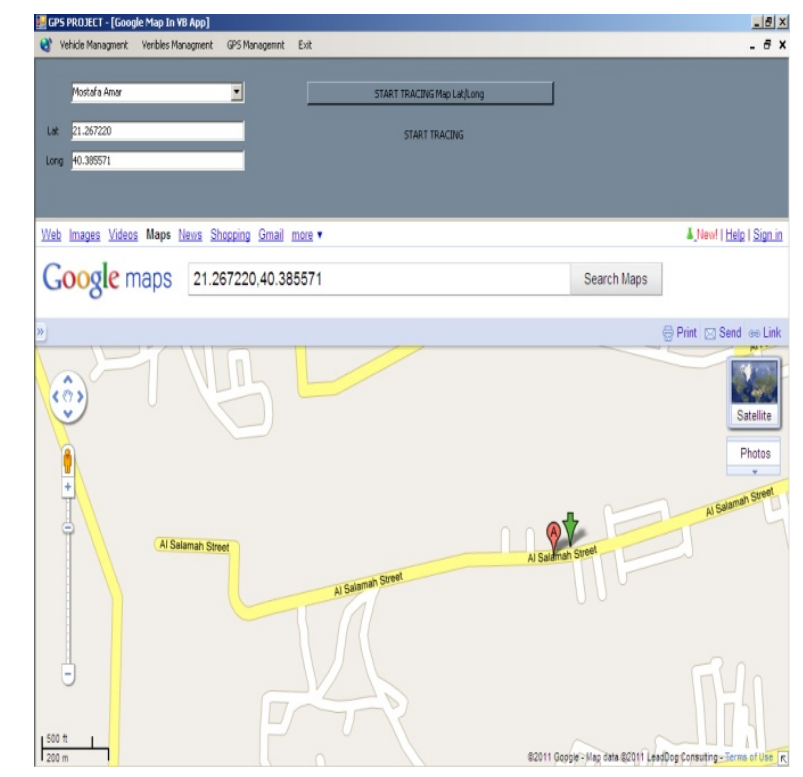

\section{Figure 12: The Design Showing Locations on Google Maps Form.}

\section{CONCLUSION}

The objective of this paper, design and implement a trucks tracking system that receives GPS reading from a GPS tool. Read data and location will be stored in the database combined with data and time. Moreover, it will also be sent to the GPS position to the station (company or owner) and the receiver will show the position on the associated map. Accuracy of the system is highly dependent on the GPS device and the coordinates received from GPS satellite while reliability and usability depend on the reliability of the mobile communications network.

Developing a semantic web based software to display all transmitted information to end user along with displaying location of vehicle on a map.GPS tracking system ability to help the fleet decrease leisure times, enhance directing operations, and better customer service. As a result, it may be able to lower overhead business costs. The semantic web is designed to be user friendly, interactive, secure, and reliable. This paper includes various features like ingenuity, simplicity of design and ease of implementation. It is completely integrated so that once it is implemented in all vehicles, then it is easy to track vehicle any time.

\section{REFERENCES}

[1] Indraneel B. , Namrata S. , Sana P. , Shalini , Sneha A. , Vitasta T., "Vehicle Tracking and Locking System Using GPS and GSM", International Advanced Research Journal in Science, Engineering and Technology, IARJSET, ISSN (Online) 2393-8021 ISSN (Print) 23941588, Vol. 4, Issue 2, February 2017.

[2] Khalifa A. S., Ibrahim M. I., "Design and Implementation of Web-Based GPS-GPRS Vehicle Tracking System", IJCSET, December, Vol 3, Issue 12, 443-448, ISSN: 2231-0711, 2013.

[3] Akintunde M. A., Stephen E. E., Edward E. D., Mohd K. R., Choi S. L., and Mkomange C. W., "The Use of Global System of Mobile Communication (GSM) Among University Students in Malaysia", International Journal of Innovation, Management and Technology, vol. 2, no. 6, pp. 547-588,Malaysia, Dec. 2011.

[4] Junaid A., Shaib N., Taha A., Naveed A. and syed N., "Implementation of GSM based Commercial Automobile Tracker Using PIC 18 F452 and Development of Google Earth Embedded Monitoring Software", IEEE International Conference on Research and Development, UPM Serdang, Malaysia, 16-18 Nov. 2009.

[5] Sana B., Umber N. and Mehreen S., "Analytical Survey for Assuring Quality Standards in GPS based Tracking System", International Journal of Computer Applications (0975 - 8887) Volume 111 - No 8, February 2015.

[6] Gautham A. S., "Google Revises Their Map, Adds Offline Version and 3D Imaging", echGau.org. Retrieved June 9, 2012.

[7] Ambade S. D. and S.A Shaikh, "Design and Implementation Of Vehicle Tracking System 
Using GPS " Journal of Information Engineering and Applications www.iiste.org ISSN 2224-5758 (print) ISSN 2224-896X (online) Vol 1, No.3, 2011.

[8] Mashood M., "GPS based Advanced Vehicle Tracking and Vehicle Control System", I.J. Intelligent Systems and Applications, Published Online February 2015 in MECS, 2015.

[9] Bhavani T., "XML Databases and the Semantic Web", Library of Congress Cataloging -inPublication Data, ISBN 0-8493-1031 -8 , 2012. [10]Abid k. and Ravi M., "GPS - GSM Based Tracking System", International Journal of
Engineering Trends and Technology- Volume3, Issue2- Page 161, ISSN: 2231-5381, 2012.

[11]Hong W., Zhenjie P., and Wentao Z., "The Design and Implementation of Information System in Civil Airport Emergency Management Based on Semantic Web", 25th Chinese Control and Decision Conference (CCDC), IEEE 2013.

[12] Valerie C. and Xueheng Hu., "Fuzzy Set and Semantic Similarity in Ontology Alignment", WCCI 2012 IEEE World Congress on Computational Intelligence, Brisbane, Australia June, 10-15, 2012.

\section{APPENDIX}

\section{- Source Code}

\section{- Showing locations on Google maps}

Imports System.Text

Public Class Form1

Private Sub btnMapLatLong_Click(ByVal sender As System.Object, ByVal e As System.EventArgs) Handles btnMapLatLong.Click

Label3.Text $=$ "START TRACING"

Timer1.Enabled $=$ True

End Sub

Private Sub Form1_FormClosed(ByVal sender As Object, ByVal e As

System.Windows.Forms.FormClosedEventArgs)

Handles Me.FormClosed

MainMenu.MapsGPSToolStripMenuItem.Enabled = True

End Sub

Private Sub Form1_Load(ByVal sender As System.Object, ByVal e As System.EventArgs) Handles MyBase.Load Load_Active_Sim()

End Sub
Sub Load Active Sim()

Dim sql As String = "SELECT drv_info.drv_name,sim_starter.sim_id FROM sim_starter LEFT JOIN vclusr_info ON

(sim_starter.vclusr_dir_id = vclusr_info.vclusr_id) LEFT JOIN drv_info ON (drv_info.drv_id = vclusr_info.usr_id) WHERE sim_starter.status=1"

MessageBox.Show(sql)

sCommand $=$ New SqlCommand(sql, SQLConn)

sAdapter $=$ New SqlDataAdapter(sCommand $)$

sBuilder $=$ New SqlCommandBuilder(sAdapter)

sDs $=$ New DataSet ()

sAdapter.Fill(sDs, "vclusr_info")

sTable $=$ sDs.Tables("vclusr_info")

ComboBox1.DataSource $=$ sTable

ComboBox1.DisplayMember = "drv_name"

ComboBox1.ValueMember $=$ "sim_id"

End Sub

Private Sub Timer1_Tick(ByVal sender As System.Object, ByVal e As System.EventArgs) Handles Timer1.Tick 
International Journal of Trend in Scientific Research and Development, Volume 1(4), ISSN: 2456-6470 www.ijtsrd.com

Dim SQL As String = ""

$\mathrm{SQL}=$ "SELECT * FROM sim_starter WHERE status $=0 "$

Dim DR1 As SqlDataReader $=$ SQLExecuteReader(SQL)

If Not DR1.HasRows Then

txtLat.Clear()

txtLong.Clear()

Timer1.Enabled $=$ False

Label3.Text $=$ "STOPED TRACING"

Exit Sub

End If

If ComboBox1.SelectedValue $>0$ Then

$\mathrm{SQL}=$ "SELECT

sim_dir_loc.sim_dir_id,dirictions_locations.dir_lat,dir ictions_locations.dir_long from sim_dir_loc LEFT JOIN dirictions_locations ON (sim_dir_loc.loc_id = dirictions_locations.loc id) WHERE

sim_dir_loc.sim_id $="+$

ComboBox1.SelectedValue.ToString + " ORDER BY sim_dir_loc.loc_id DESC"

Dim DR As SqlDataReader $=$ SQLExecuteReader(SQL)

If DR.HasRows Then

$$
\text { DR.Read() }
$$$$
\text { txtLat.Text = DR("dir_lat").ToString }
$$$$
\text { txtLong.Text = DR("dir_long").ToString }
$$

Else

txtLat.Clear()

txtLong.Clear()
Timer1.Enabled $=$ False

Label3.Text $=$ "STOPED TRACING"

Exit Sub

End If

\section{End If}

If txtLat.Text $=$ String.Empty Or txtLong.Text $=$ String.Empty Then

MessageBox.Show("Supply a latitude and longitude value.", "Missing Data")

End If

Try

Dim lat As String = String.Empty

Dim lon As String = String.Empty

Dim queryAddress As New StringBuilder() queryAddress.Append("http://maps.google.com/maps $? \mathrm{q}=")$

' build latitude part of query string

If txtLat.Text $<>$ String.Empty Then

$$
\text { lat }=\text { txtLat.Text }
$$

$$
\text { queryAddress.Append(lat + "\%2C") }
$$

End If

' build longitude part of query string

If txtLong. Text $<>$ String.Empty Then

$$
\begin{aligned}
& \text { lon }=\text { txtLong. Text } \\
& \text { queryAddress.Append(lon) }
\end{aligned}
$$

End If

webBrowser1.Navigate(queryAddress.ToString()) 
International Journal of Trend in Scientific Research and Development, Volume 1(4), ISSN: 2456-6470 www.ijtsrd.com

Catch ex As Exception

MessageBox.Show(ex.Message.ToString(), "Error")

End Try

End Sub

End Class

\section{- SQL Functions}

Module SQL_Functions

Public FCH

Public sCommand As SqlCommand

Public sAdapter As SqlDataAdapter

Public sBuilder As SqlCommandBuilder

Public sDs As DataSet

Public sTable As DataTable

Dim SQLConnectionString As String =

My.Settings.Default.ConnectionString

Public Function SQLConn() As SqlConnection

SQLConn $=$ New SqlConnection

If SQLConn. State $=$ ConnectionState.Closed Then

SQLConn.ConnectionString =

SQLConnectionString

Try

$$
\text { SQLConn.Open() }
$$

Catch exError As SqlException

MsgBox(exError.Message)

End

End Try

End If
End Function

Public Function GetData(ByVal sqlCommand As String) As DataTable

Dim command As New

SqlCommand(sqlCommand, SQLConn)

Dim adapter As SqlDataAdapter $=$ New SqlDataAdapter()

Dim Table As New DataTable

adapter.SelectCommand $=$ command

adapter.Fill(Table)

'SQLConn.Close()

Return Table

End Function

Public Function ExecuteSQL(ByVal sqlCommand As String) As Integer

Dim command As New

SqlCommand(sqlCommand, SQLConn)

'command.ExecuteNonQuery()

Try

ExecuteSQL = command.ExecuteScalar()

Catch exError As SqlException

MsgBox(exError.Message)

End

End Try

'SQLConn.Close()

End Function

Public Function SQLExecuteReader(ByVal sqlcommand As String) As SqlDataReader

Dim CMD As New SqlCommand(sqlcommand, SQLConn) 
Dim DR As SqlDataReader = CMD.ExecuteReader()

'SQLConn.Close()

Return DR

End Function

Public Function myExecuteScalar(ByVal sqlcommand As String) As Integer

Dim CMD As New SqlCommand(sqlcommand, SQLConn)

Dim ES As Integer

If IsDBNull(CMD.ExecuteScalar()) = False Then

$$
\text { ES = Convert.ToInt32(CMD.ExecuteScalar()) }
$$

Else

$$
\mathrm{ES}=0
$$

End If

SQLConn.Close()

Return ES

End Function

End Module 